BULLETIN OF THE

AMERICAN MATHEMATICAL SOCIETY

Volume 77, Number 4, July 1971

\title{
MULTIPLICITY FORMULAS FOR CERTAIN SEMISIMPLE LIE GROUPS
}

\author{
BY J. LEPOWSKY 1 \\ Communicated by Louis Auslander, December 14, 1970
}

1. Introduction. The main purpose of this note is to announce a result (Theorem 5) concerning finite-dimensional representations of semisimple Lie groups of real rank 1 . Theorem 5 extends [5, Corollary 3.8], which states that the finite-dimensional spherical representations are the conical ones, and [7, Corollary 1 of Theorem 2.1], which asserts the existence of minimal types for finite-dimensional representations of complex groups. Our method, based on a previously unpublished general formula (see $\$ 2$ ) due to B. Kostant, yields several other multiplicity results as well.

Let $H_{1}$ be a real Lie group and let $H_{2}$ be a Lie subgroup of $H_{1}$. Let $\alpha \in \hat{H}_{1}$ ( - denotes the set of equivalence classes of finite-dimensional continuous complex irreducible representations), and assume that the restriction to $H_{2}$ of any member of $\alpha$ splits into a direct sum of irreducible representations of $H_{2}$. For all $\beta \in \hat{H}_{2}$, let $m(\alpha, \beta)$ denote the corresponding multiplicity.

We are concerned here with the case in which $H_{1}$ is a connected real semisimple Lie group $G$ of real rank 1 , and $H_{2}$ is the connected Lie subgroup $K$ corresponding to $\mathfrak{f}$, where $\mathfrak{g}=\mathfrak{l}+\mathfrak{p}$ is a Cartan decomposition of the Lie algebra of $G$. The solution of the problem of computing the multiplicities for the pair $(G, K)$ is contained in the solution of the problem for the "dualized" pair $\left(U_{1}, U_{2}\right)$. Here $U_{1}$ is the simply connected compact Lie group with Lie algebra $+i p \subset g_{C}$ (the complexification of $\mathfrak{g}$ ), and $U_{2}$ is the connected compact Lie subgroup of $U_{1}$ corresponding to $\mathrm{l}$.

It is well known (see [4, Chapter IX] for the notation and classification) that if the Lie algebra of $U_{1}$ is assumed simple, there are five possibilities for the pair $\left(U_{1}, U_{2}\right)$ :

$$
\begin{array}{ll}
\text { Type } \mathrm{A}_{n}:\left(\mathrm{SU}(n+1), \mathrm{S}\left(U_{1} \times U_{n}\right)\right) & \text { (special unitary case) } \\
\text { Type } \mathrm{B}_{n}:(\operatorname{Spin}(2 n+1), \operatorname{Spin}(2 n)) & \text { (orthogonal case) }
\end{array}
$$

AMS 1970 subject classifications. Primary 17B10, 20G05; Secondary 05A99, 17 B25.

Key words and phrases. Rank 1 semisimple Lie groups, finite-dimensional representations, multiplicity formulas, symplectic groups, $F_{4}$, Kostant's multiplicity formula, class 1 representations.

1 The results announced here are contained in the author's Ph.D. thesis, written under the guidance of Professor B. Kostant and Professor S. Helgason at M.I.T., where the author held an N.S.F. Graduate Fellowship. 


$$
\begin{array}{ll}
\text { Type } \mathrm{C}_{n}:(\operatorname{Sp}(n), \operatorname{Sp}(1) \times \operatorname{Sp}(n-1)) & \text { (symplectic case) } \\
\text { Type } \mathrm{D}_{n}:(\operatorname{Spin}(2 n), \operatorname{Spin}(2 n-1)) & \text { (orthogonal case) } \\
\text { Type } \mathrm{F}_{4}:\left(\mathrm{F}_{4}, \operatorname{Spin}(9)\right) & \text { (exceptional case) }
\end{array}
$$

The multiplicity formulas for the special unitary and orthogonal cases are well known and classical (see [1]). Starting from Kostant's formula ( $\$ 2$, and using combinatorial reasoning, we can easily recover these formulas, as well as obtain (by much harder arguments) a formula for the symplectic case $(\S 3)$ and partial formulas for the exceptional case ( $\$ 4)$. Our results stated in $\$ 3$ and $\$ 4$ seem to be new, although G. C. Hegerfeldt [3] has obtained a formula for certain other pairs of symplectic groups. Our symplectic result is expressed rather interestingly in terms of the combinatorial function $F_{m}$ defined below. It would be desirable to have a complete multiplicity formula for the exceptional case. In $\$ 5$ we state Theorem 5 and another application of our results.

2. Kostant's formula. Let $U$ be a compact connected Lie group and $V$ a compact connected Lie subgroup of $U$. Let $S$ and $T$ be maximal tori of $U$ and $V$, resp., such that $T \subset S$. We denote by $\mathfrak{u}$ the Lie algebra of $U$, and by $\mathfrak{b}, \mathfrak{z}$ and $\mathrm{t}$ the Lie subalgebras of $\mathfrak{t}$ corresponding to $V, S$ and $T$, resp., so that $t \subset \xi$. Let $\nu \rightarrow \nu^{*}$ denote the restriction map from $\mathfrak{B}^{\prime}$ to $\mathfrak{t}^{\prime}$, where $\mathfrak{B}^{\prime}=\operatorname{Hom}_{R}(\mathbb{B}, \quad C)$ and $\mathrm{t}^{\prime}=\operatorname{Hom}_{R}(\mathrm{t}, C)$.

Assumption. We assume that $\mathfrak{b}$ contains a regular element of $\mathfrak{u}$. We may choose an element $X \in \mathfrak{t}$ which is regular in both $\mathfrak{u}$ and $\mathfrak{v}$. We fix the unique Weyl chambers in $s$ and $t$ (for $\mathfrak{u}$ and $\mathfrak{b}$, resp.) which contain $X$. Positivity and dominance of roots and weights are taken with respect to these chambers.

Let $\omega_{1}, \cdots, \omega_{r} \in \mathrm{t}^{\prime}$ be the positive weights of the canonical representation of $\mathfrak{b}$ on $(\mathfrak{u} / \mathfrak{b})_{C}$, repeated according to multiplicity if necessary. For every $\mu \in \mathfrak{t}^{\prime}$, let $P(\mu)$ be the number of nonnegative integral $r$-tuples $n_{1}, \cdots, n_{r}$ such that $\mu=\sum_{i=1}^{n} n_{i} \omega_{i}$.

Let $\rho \in \mathfrak{B}^{\prime}$ be half the sum of the positive roots of $\mathfrak{u}$, and let $W$ be the Weyl group of $\mathfrak{t}$, regarded as a group of linear transformations of $\mathcal{B}^{\prime}$. Let $D_{U} \subset \mathcal{B}^{\prime}$ and $D_{V} \subset t^{\prime}$ denote the sets of dominant integral linear forms for $U$ and $V$, respectively. We identify $\hat{U}$ with $D_{U}$ and $\hat{V}$ with $D_{V}$ by assigning to each equivalence class of representations the highest weight of any of its members.

Theorem 1 (Kostant). For all $\lambda \in D_{U}$ and $\mu \in D_{V}$, we have

$$
m(\lambda, \mu)=\sum_{\sigma \in W}(\operatorname{det} \sigma) P\left((\sigma(\lambda+p))^{*}-\left(\mu+\rho^{*}\right)\right) .
$$


Kostant has shown that a modified version of Theorem 1 remains true when the above assumption is dropped. Theorem 1 is easily proved from Weyl's character formula by generalizing the proof of the special case of Theorem 1 given in [2].

3. The symplectic case. Let $n=2,3, \cdots$, and let $U=\operatorname{Sp}(n)$ ' $V=\operatorname{Sp}(1) \times \operatorname{Sp}(n-1)$, so that $z=t$ in the above notation. Now $\mathfrak{B}^{\prime}$ has a real form with a basis $\left\{\phi_{1}, \cdots, \phi_{n}\right\}$ such that the roots of $\mathfrak{u}_{C}$ with respect to its Cartan subalgebra $\AA_{C}$ are $\pm \phi_{i} \pm \phi_{j}(1 \leqq i<j \leqq n)$ and $\pm 2 \phi_{i}(1 \leqq i \leqq n)$, and the roots of $\mathfrak{v}_{\mathrm{C}}$ with respect to $\mathfrak{B}_{C}$ are $\pm \phi_{i} \pm \phi_{j}$ $(2 \leqq i<j \leqq n)$ and $\pm 2 \phi_{i}(1 \leqq i \leqq n)$. We may take

$$
\begin{aligned}
& D_{U}=\left\{\sum_{i=1}^{n} a_{i} \phi_{i} \mid a_{i} \in Z, a_{1} \geqq \cdots \geqq a_{n} \geqq 0\right\}, \\
& D_{V}=\left\{\sum_{i=1}^{n} b_{i} \phi_{i} \mid b_{i} \in Z, b_{1} \geqq 0, b_{2} \geqq b_{3} \geqq \cdots \geqq b_{n} \geqq 0\right\} .
\end{aligned}
$$

Definition. Let $l, m \in \boldsymbol{Z}, m \geqq 1$, and let $q_{1}, q_{2}, \cdots, q_{m} \in \boldsymbol{Z}_{+}$. We define $F_{m}\left(l ; q_{1}, q_{2}, \cdots, q_{m}\right)$ to be the number of ways of putting $l$ indistinguishable balls into $m$ distinguishable boxes with capacities $q_{1}, q_{2}, \cdots, q_{m}$.

Theorem 2. Let $\lambda=\sum_{i=1}^{n} a_{i} \phi_{i} \in D_{U}$ and $\mu=\sum_{i=1}^{n} b_{i} \phi_{i} \in D_{V}$. Define

$$
\begin{aligned}
& A_{1}=a_{1}-\max \left(a_{2}, b_{2}\right), \\
& A_{i}=\min \left(a_{i}, b_{i}\right)-\max \left(a_{i+1}, b_{i+1}\right) \quad(2 \leqq i \leqq n-1), \\
& A_{n}=\min \left(a_{n}, b_{n}\right) .
\end{aligned}
$$

Then $m(\lambda, \mu)=0$ unless $b_{1}+\sum_{i=1}^{n} A_{i} \in 2 \boldsymbol{Z}$ (that is, $\sum_{i=1}^{n}\left(a_{i}+b_{i}\right) \in 2 \boldsymbol{Z}$ ) and $A_{1}, A_{2}, \cdots, A_{n-1} \geqq 0$ ( $A_{n} \geqq 0$ automatically). Under these conditions,

$$
\begin{aligned}
m(\lambda, \mu)= & \sum_{L \subset\{1,2, \cdots, n\}}(-1)^{|L|} \\
& \cdot\left\{\begin{array}{c}
\left.n-2-|L|+\frac{1}{2}\left(-b_{1}+\sum_{i=1}^{n} A_{i}\right)-\sum_{i \in L} A_{i}\right) \\
n-2
\end{array}\right) \\
= & F_{n-1}\left(\frac{1}{2}\left(b_{1}-A_{1}+\sum_{i=2}^{n} A_{i}\right) ; A_{2}, A_{3}, \cdots, A_{n}\right) \\
& -F_{n-1}\left(\frac{1}{2}\left(-b_{1}-A_{1}+\sum_{i=2}^{n} A_{i}\right)-1 ; A_{2}, A_{3}, \cdots, A_{n}\right),
\end{aligned}
$$


where $|L|$ denotes the number of elements in $L$, and $\left(\begin{array}{l}x \\ y\end{array}\right)$ denotes the binomial coefficient, which is defined to be 0 if $x<y$.

4. The exceptional case. Let $U=\mathrm{F}_{4}, V=\operatorname{Spin}(9)$, so that again $\mathfrak{B}=\mathrm{t}$. There is a real form of $\mathfrak{g}^{\prime}$ with a basis $\left\{\psi_{1}, \psi_{2}, \psi_{3}, \psi_{4}\right\}$ such that the roots of $\mathfrak{u}_{C}$ with respect to $\mathfrak{B}_{C}$ are $\pm \psi_{i} \pm \psi_{j}(1 \leqq i<j \leqq 4), \pm \psi_{i}$ $(1 \leqq i \leqq 4)$ and $\frac{1}{2} \sum_{i=1}^{4} \pm \psi_{i}$, and the roots of $\mathfrak{b}_{C}$ with respect to $\varepsilon_{C}$ are $\pm \psi_{i} \pm \psi_{j}(1 \leqq i<j \leqq 4)$ and the elements $\frac{1}{2} \sum_{i=1}^{4} \pm \psi_{i}$ with an odd number of minus signs. We may take

$$
\begin{array}{r}
D_{U}=\left\{\sum_{i=1}^{4} a_{i} \psi_{i} \mid \text { either } a_{i} \in Z(1 \leqq i \leqq 4) \text { or } a_{i} \in Z+\frac{1}{2}(1 \leqq i \leqq 4),\right. \\
\left.a_{1} \geqq a_{2} \geqq a_{3} \geqq a_{4} \geqq 0, a_{1} \geqq a_{2}+a_{3}+a_{4}\right\}, \\
D_{V}=\left\{\sum_{i=1}^{4} b_{i} \psi_{i} \mid \text { either } b_{i} \in \boldsymbol{Z}(1 \leqq i \leqq 4) \text { or } b_{i} \in \boldsymbol{Z}+\frac{1}{2}(1 \leqq i \leqq 4),\right. \\
\left.b_{1} \geqq b_{2} \geqq b_{3} \geqq\left|b_{4}\right|, b_{1} \geqq b_{2}+b_{3}+b_{4}\right\} .
\end{array}
$$

Theorem 3. Let $a \in \boldsymbol{Z}_{+}$, so that $\lambda=a \psi_{1} \in D_{U}$. Let $\mu=\sum_{i=1}^{4} b_{i} \psi_{i} \in D_{V}$. Then $m(\lambda, \mu)=1 \Leftrightarrow b_{2}=b_{3}=-b_{4}$ and $b_{1}+b_{2} \leqq a$; otherwise, $m(\lambda, u)=0$.

THEOREM 4. Let $\lambda=\sum_{i=1}^{4} a_{i} \psi_{i} \in D_{U}$. Then $\mu=a_{2} \psi_{1}+a_{3} \psi_{2}+a_{4} \psi_{3}$ $-a_{4} \psi_{4} \in D_{V}$, and $m(\lambda, \mu)=1$.

5. Applications. Let $G=K A N$ be an Iwasawa decomposition of a connected real semisimple Lie group of real rank 1 , and let $M$ be the centralizer of $A$ in $K$. For all $\alpha \in \hat{G}$, let $\gamma(\alpha) \in \hat{M}$ be the class under which the highest restricted weight space of any member of $\alpha$ transforms. Using Theorems 2 and 4 and the known multiplicity formulas for the other rank 1 simple groups, we can prove:

THEOREM 5. For all $\gamma \in \hat{M}$ there exists $\beta(\gamma) \in \hat{K}$ satisfying $m(\beta(\gamma), \gamma)$ $=1$, such that $m(\alpha, \beta(\gamma(\alpha)))=1$ for all $\alpha \in \hat{G}$.

The correspondences $\gamma \rightarrow \beta(\gamma)$ and $\alpha \rightarrow \beta(\gamma(\alpha))$ can be interpreted geometrically in terms of walls of Weyl chambers. Using this interpretation, we can construct analogues for rank 1 groups of the homomorphisms given in [7, Theorems 2.2 and 2.3].

An element $\alpha \in \hat{G}$ is said to be of class 1 if it contains the trivial element of $\hat{K}$. Theorems 2 and 3 , together with known facts about the other rank 1 simple groups, yield:

Theorem 6. If $\alpha \in \hat{G}$ is of class 1 , then $m(\alpha, \beta) \leqq 1$ for all $\beta \in \hat{K}$. 
Theorem 6 is essentially the same as a result [6, Theorem 6] recently obtained by Kostant by different methods. Our treatment, however, gives new information-an explicit list of the highest weights of the elements of $\hat{K}$ contained in a given class 1 element of $\hat{G}$.

Formula (1) reduces Theorem 5 in the symplectic case to the fact that the number of ways of putting 0 balls into boxes is 1 , and Theorem 6 to the fact that the number of ways of putting balls in 1 box of finite capacity is $\leqq 1$.

\section{REFERENCES}

1. H. Boerner, Darstellungen von Gruppen mit Berücksichtigung der Bedürfnisse der modernen Physik, Springer-Verlag, Berlin, 1955; English transl., North-Holland, Amsterdam; Interscience, New York, 1963. MR 17, 710; MR 26 \#6272.

2. P. Cartier, On H. Weyl's character formula, Bull. Amer. Math. Soc. 67 (1961), 228-230. MR 26 \#3828.

3. G. C. Hegerfeldt, Branching theorem for the symplectic groups, J. Mathematical Phys. 8 (1967), 1195-1196. MR 37 \#339.

4. S. Helgason, Differential geometry and symmetric spaces, Pure and Appl. Math., vol. 12, Academic Press, New York, 1962. MR 26 \#2986.

5. - - A duality for symmetric spaces with applications to group representations, Advances in Math. 5 (1970), 1-154.

6. B. Kostant, On the existence and irreducibility of certain series of representations, Bull. Amer. Math. Soc. 75 (1969), 627-642. MR 39 \#7031.

7. K. R. Parthasarathy, R. Ranga Rao and V. S. Varadarajan, Representations of complex semi-simple Lie groups and Lie algebras, Ann. of Math. (2) 85 (1967), 383429. MR 37 \#1526.

Brandeis University, Waltham, Massachusetts 02154

Massachusetts Institute of Technology, Cambridge, Massachusetts 02139 